\title{
Detection of Cardiac Disease from ECG record using Regional Convolutional Neural Network
}

\author{
Miss. Swati Dilip Thakare, Prof. Santosh Kumar
}

\begin{abstract}
Electrocardiogram (ECG) demonstrates the electrical activities in the heart, and is the most important physiological parameter that gives the proper analysis regarding the functioning of the heart. In this work, an automatic and powerful deep feature learning process is used. By using it a convolution neural network (CNN) is exert to study pristine features from the raw ECG data to achieve disease identification without any complex feature engineering process. An Electrocardiogram (ECG) is the primary diagnostic tool for recording and interpreting ECG signals. These signals holds details regarding different kinds of arrhythmias, ECG signals are complex and non-linear in nature so that it is tough to analyse these signals manually. Furthermore, the exposition of ECG signals is subjective and may vary from expert to expert. Therefore, a computer aided diagnosis (CAD) system has been proposed, which guarantees that the evaluation of ECG signals is objective and reliable. In proposed system, a convolution neural network (CNN) technology is used to automatically detect different ECG segments. An efficient electrocardiogram (ECG) arrhythmia classification technique with deep 11 layer convolution neural network (CNN) is used in this system. In which every ECG signal will transform into a 2D gray-scale image as an input to the $\mathrm{CNN}$ classifier. Batch normalization, Xavier initialization, data augmentation and dropout are different deep learning techniques which are used for CNN optimization.
\end{abstract}

Index Terms - Arrhythmia, Atrial fibrillation, Atrial flutter, Convolution neural network, Deep Learning, Electrocardiogram signals, Ventricular fibrillation.

\section{INTRODUCTION}

The heart diseases are the main reason for the human death. As per the survey of human death, every year, nearby 7.4 million deaths are due to non-proper functioning of heart. Out of which, $52 \%$ of deaths due to strokes and $47 \%$ deaths due to coronary heart diseases. So, it is necessary to identify different heart disease at an initial stage to protect heart-related deaths [14].

Manuscript revised on November 15, 2019 and published on November 26, 2019

Miss. Swati Dilip Thakare, PG Student, Computer Engineering Department, SITRC, Nashik-422213, India

Prof. Santosh Kumar, Assistant Professor, Computer Engineering Department, SITRC, Nashik-422213, India.
Heart is the most crucial organ of human body. As per the survey of World Health Organization (WHO) cardiovascular disease (CVD) is the primary disease in the age group of 30-60[14]. In India among all the diseases, cardiovascular diseases are the primary reason, which cause more people to die every year [12]. We know that heart attack occurs suddenly without any indication but the disturbances in heart activities may found before it. As we grow, the cardiovascular system weakens and become more subjective to disease [7].

An arrhythmia depicts an irregularity in heartbeat - the heart pulses may too slowly, too fast, or randomly. Arrhythmias occur once the electrical signals to heart that manage heart pulses are not operating properly [10]. Sometimes, we observe random heart pulses, which may feel like a racing heart or fluttering. Several heart arrhythmias are not dangerous; but, if they're notably unusual, or occur from a weak or broken heart, arrhythmias will cause serious and even doubtless fatal symptoms. Heart pulse rate of healthy person lies between 60 to $100 \mathrm{bpm}$ when resting. There are several types of arrhythmia from them Atrial fibrillation (Afib), Atrial flutter (Afl) and Ventricular fibrillation (Vfib) are the common occurring types of arrhythmia [6].

Here, we mainly concentrate the object-centred scenario. To enhance the nature of ROIs and recoup the picture without obscuring curios, we proposed to encode the main regions alongside some background features, i.e., quantized shading histogram and nearby descriptors. With that, we always try to maintain visual quality of that object region. Then again, the bit-rate utilization can be additionally diminished with encoded quantized background features. In our methodology, we select a set of available images as prior and try to achieve background synthesis without semantic distortion.

\section{SYSTEM ARCHITECTURE}

\section{A. Problem Statement}

To design and develop automatic and robust deep feature learning process using a Faster Regional Convolution Neural Network (R-CNN) and to learn inherent features from the raw ECG signal to perform disease detection without having any complex feature engineering process.

\section{B. System Overview}

Our CNN based ECG arrhythmia classification consists following steps: ECG data pre-processing, and the ECG arrhythmia classifier.

In our system, for training and testing of CNN model we are going to use the arrhythmia database. This model handles two-dimensional image as an input data. ECG signals are converted into ECG images for the ECG data pre-processing. 
In CNN classifier step the classification of ECG types are take place.

\section{Data flow}

Our system includes following steps: ECG data pre-processing, and the ECG arrhythmia classifier. In this system, For training and testing of CNN model we used the MIT-BIH arrhythmia database. During ECG data pre-processing step, ECG signal is converted into 2D ECG image, and this image is used for classification of ECG types which is carried out in CNN classifier step. Following Fig Shows overall working of propose architecture.

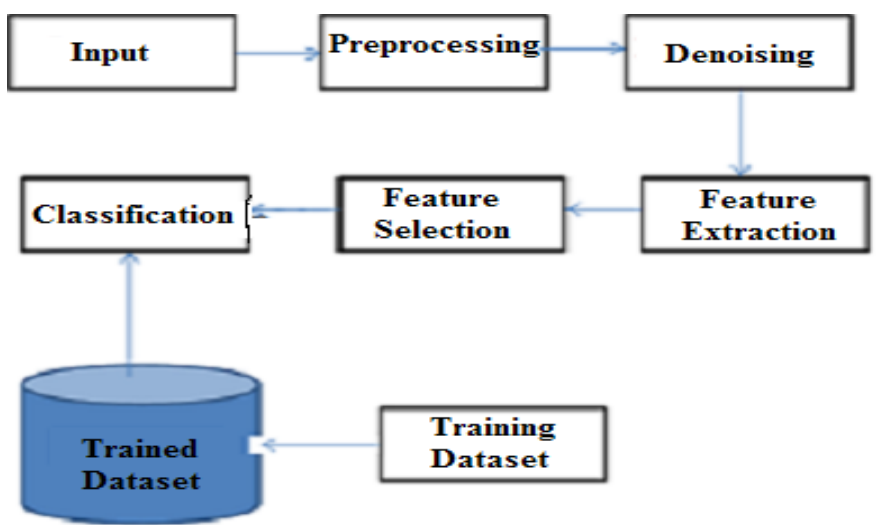

Fig. 1. Workflow of system

\section{Preprocessing:}

The raw ECG data sr(n) is separated to emphasize the QRS segment, which is distinguished by a excessive slope. The distinct cardiogram signal is acquired by creating subtraction between adjacent samples.

\section{DE noising:}

Because of preprocessing, signals are polished and made ready for actual processing. Removal of unwanted noise is one of the preprocessing units. The Power-line causes electromagnetic fields which are said to be common noise source for an ECG Signal. These are characterized by the sinusoidal interference of $50-60 \mathrm{~Hz}$ accompanied with a various harmonic.

\section{Segmentation:}

The ECG signals are fragmented and classified according to cardiac conditions of heart, and the prescribed annotations are retrieved from a public database.

\section{Feature Extraction:}

In this process different features of an ECG signal are extracted. This process is followed by feature selection to select only important features for classification process. We did not follow the traditional process of automated CAD systems.

\section{Classification:}

Convolutional Neural Networks (CNNs) is advancement to neural networks in which convolutional layers replace with sub-sampling layers, redolent of simple and complicated cells in the human cortical area, that are frequently utilized for the purpose of "deep learning" like object recognition in large image archives during achieving the modern performances.

\section{D.Algorithm}

The algorithm used for proposed framework is Faster R-CNN. The main motivation behind use of this technique is that instead of running a CNN 2,000 times per image, we can run it just once per image and get all the regions of interest (regions containing some object) [2] [7].

Faster R-CNN mainly used for object detection instead of pattern matching. It is the updated version of Fast R-CNN. The fundamental distinction between both of them is that Fast RCNN uses selective search for generating different Regions of Interest, while Faster RCNN uses "Region Proposal Network" (RPN) to generate different Regions Of Interest. The input to RPN is image feature maps and output of it is a collection of object proposals with an object-ness score. Faster R-CNN has two networks:

1. Region Proposal Network (RPN) used for generating region proposals

2. Network which is used to detect object using above generated proposals.

Faster R-CNN Algorithm Steps:

1. Take the per-trained Convolution Neural Networks (CNN).

2. Retrained this model by training the last layer of the network based on the number of classes that need to be identified.

3. Pass input image to the retrained trained convolutional neural network. It will return the feature map for input image.

4. Apply the Region proposal network on these feature maps. This gives the object proposals with their score of object-ness.

5. Apply a RoI pooling layer on these proposals to make all the proposals to the equal size.

6. Finally, pass the proposals to a fully connected layer which has two layer one is softmax layer and other is linear regression layer at its top, to classify and output the bounding boxes for objects.

\section{PROBLEM FORMULATION}

\section{A. Mathematical Model}

Let the system be described by $\mathrm{S}$,

$\mathrm{S}=\{\mathrm{I}, \mathrm{F}, \mathrm{O}\}$

Where,

$\mathrm{I}=\mathrm{I}$ is the set of input to system, $\mathrm{I}=\{\mathrm{I} 1, \mathrm{I} 2\}$

Input to system is the file containing ECG signal.

$\mathrm{F}=\mathrm{F}$ is the set of different function that system will do, $\mathrm{F}=$ $\{\mathrm{F} 1, \mathrm{~F} 2, \mathrm{~F} 3, \mathrm{~F} 4, \ldots \mathrm{Fn}\}$

Following are the different functions that are used in system.

F1: Signal Processing.

F2: DE noising.

F3: Segmentation

F4: Feature Extraction.

F5: Feature Selection 
F6: Classification

$\mathrm{O}=\mathrm{O}$ is the set of output, $\mathrm{O}=\{\mathrm{O} 1, \mathrm{O} 2, \mathrm{O} 3, \ldots \mathrm{On}\}$

Output is the class of arrhythmia.

\section{B. System Implementation}

To developed system we used windows platform. Hardware and software requirement for the developed framework is as follows:

\section{Hardware Requirement-}

- Input devices: Keyboard, Mouse, Monitor

- Processor: Pentium Processor Dual core of $2.66 \mathrm{GHz}$

- Hard disk: $500 \mathrm{~GB}$

- RAM: $1 \mathrm{~GB}$

\section{Software Requirements}

- Operating System: Windows XP /7 onwards

- Platform: Visual Studio Tool

- supporting languages: C\# Language

\section{UML Diagram}

1. Sequence diagram- In our system User, System and Dataset are the actors which defining the separate lifeline to perform operations. First user gives input to the system and then system will carry all remaining task such as data pre-processing, Denoising, feature extraction and feature selection sequentially. After giving input to system, system uses Dataset to perform these tasks and at the end it will return the result back to user.

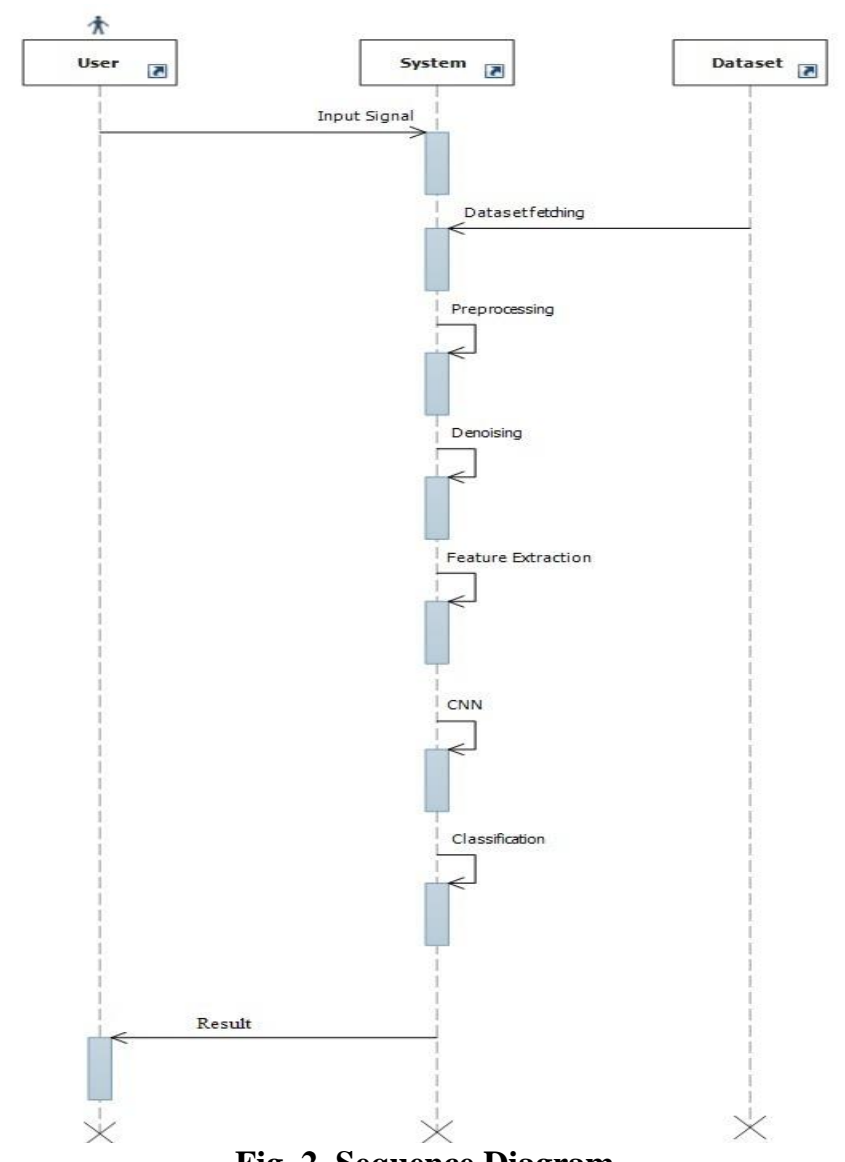

Fig. 2. Sequence Diagram
2. Class Diagram: The class diagram is a static diagram It represents the static view of a system. Class diagrams are not only used for visualization, description and recording of different aspects of the system, but also used to build executable code for the software application. In the class diagram shown below there are six classes which having separate functions and operations. The class diagram shows the relation between other classes and components such as Input and System and various such classes and their relationships are shown. The Result is shown by the show result class. In the scenario, the input is given to system. The system will do the Pre-processing, Feature Extraction, Feature Selection internally. Outputs of these classes are given to $\mathrm{CNN}$ to classify the class of input. At the end result class will show the output using detectionofarrhythmia() function.

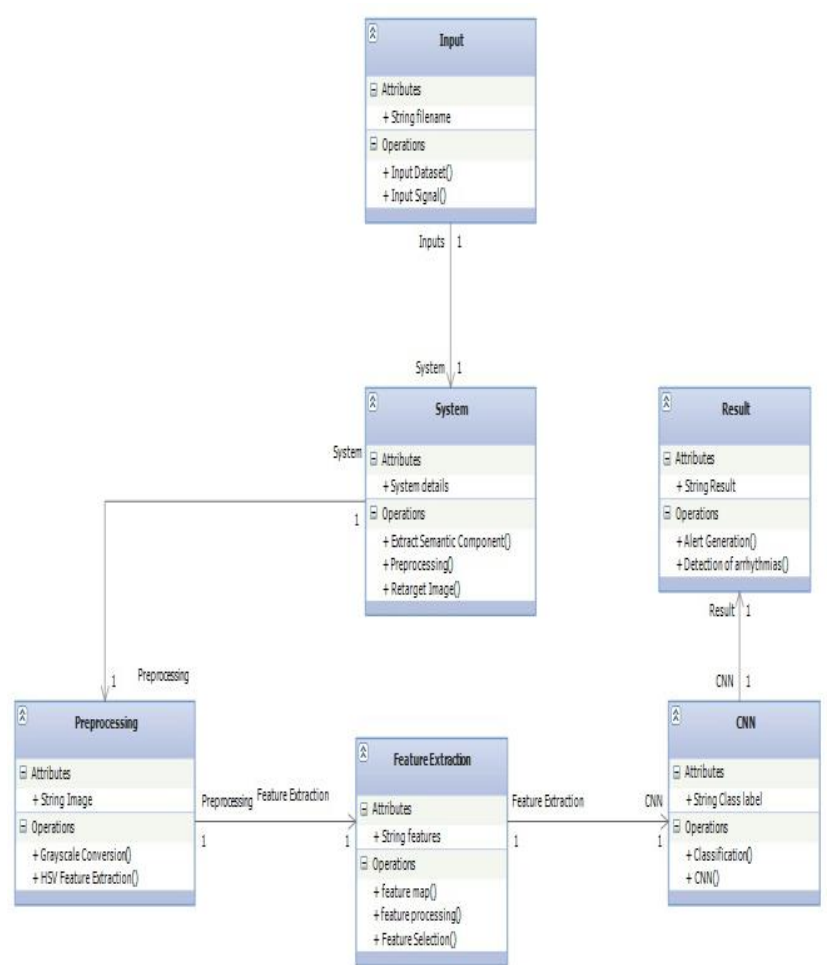

Fig. 3. Class Diagram

\section{Data Flow Diagram:}

Data Flow Diagrams (DFD) are the diagrams which show the actual flow of data in a system. The DFD shows the type of information that will be input and output from the system, how the data advances through the system, and where the data will be stored. It does not display information about the process time, nor does it show whether the process is operating in sequence or in parallel. These charts are presented at different levels. Basic level 0 shows the surface system in a single system view. Thus, the starting level represents the most basic representation of the data stream. As DDF levels increase, it provides more and more detailed views of the system. A more detailed description clearly shows the flow of the system.

1. Data Flow Diagram 0: This is the basic level of the DFD. In proposed system, input to the system is any ECG signal. The system internally processes the input and returns 
the output. The output is nothing but the classification of ECG into different classes of arrhythmia. Flowing figure shows the graphical representation of DFD 0 of the proposed system

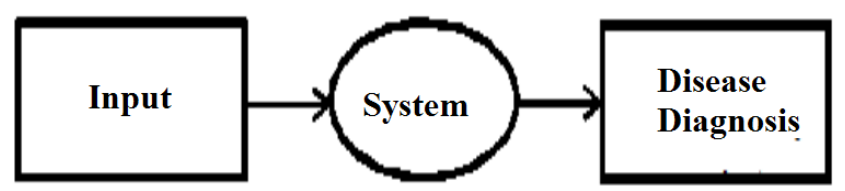

Fig. 4. DFD Level 0

2. Data Flow Diagram 1: DFD level 1 diagram used to show the main functions of the system. Figure shows the pictorial view of the DFD 1 of proposed system. In that system is divided into different processes and output of earlier process is input to the next process.

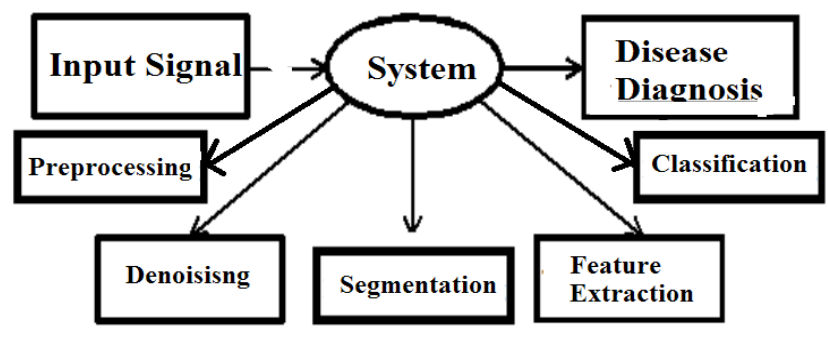

Fig. 5. DFD Level 1

\section{RESUlT}

Results of our system are depicted in terms of parameters viz. accuracy, sensitivity, specificity. These are commonly used statistics to describe a performance of any diagnostic Test in medical field. The experimental analysis is carried out on Windows 7 operating system with $\operatorname{Intel}(\mathrm{R})$ core(TM) i5 processor, 4 GB RAM. In this work, the ECG segments are not affected by time shifting and scaling thus there is no need to perform QRS detection in the pre-processing stage. Normally, the primary steps involved in analysing ECG signals are (i) filtering of noise, (ii) demonizing, (iii) Feature extraction, and (iv) Feature Selection.

\section{A. Experimental Analysis}

To implement this system, we have used Aforge an Accord dll for image feature extraction and feature selection. For testing we have used core-i5 system with 4 GB RAM. This system is build using Aforge libraries and C\# language.

\section{B. Dataset}

In our system the ECG signals were obtained from a publicly available arrhythmia database. We have obtained Vfib (Ventricular Fibrillation) ECG signals from Creighton University ventricular tachyarrhythmia, Afib (Atrial Fibrillation) and Afl (Atrial Flutter) ECG signals from MIT-BIH atrial fibrillation, and Afib (Atrial Fibrillation), Afl (Atrial Flutter), and Nsr (Normal Sinus Rhythm) ECG signals from MIT-BIH arrhythmia database. In this work, we have used lead II ECG signals. The details of the ECG signals used in this study is, we have used two different durations of ECG segments (two seconds and five seconds) in this work. The total number of ECG segments used for net A (two seconds) and net B (five seconds) is 21,709 and 8683 respectively.

\section{Evaluation Parameters}

The evaluation parameters used in the proposed system are accuracy, sensitivity, specificity.

D. Performance Analysis

We have tested our system on different dataset MIT-BIH arrhythmia database. Different Image files from dataset are given as an input to the system. We calculate accuracy, sensitivity, specificity for the ECG Images.

E. Result of proposed system

To test performance of our system we randomly select 30 ECG images from the various datasets and feed these as input to the system. From the output of our classifier we prepared following confusion matrix.

\begin{tabular}{|l|c|c|}
\hline $\mathrm{N}=30$ & Predicted No & Predicted Yes \\
\hline Actual No: & 4 & 1 \\
\hline Actual Yes: & 1 & 24 \\
\hline
\end{tabular}

From above confusion matrix we have calculated our system performance in terms of system accuracy, sensitivity, specificity is as follows.

\begin{tabular}{|l|l|l|}
\hline $\begin{array}{l}\text { Performance } \\
\text { Parameter }\end{array}$ & $\begin{array}{l}\text { Existing } \\
\text { System }\end{array}$ & $\begin{array}{l}\text { proposed } \\
\text { System }\end{array}$ \\
\hline Accuracy & 92.5 & 93.3 \\
\hline Sensitivity & 95.09 & 96 \\
\hline Specificity & 93.13 & 100 \\
\hline
\end{tabular}

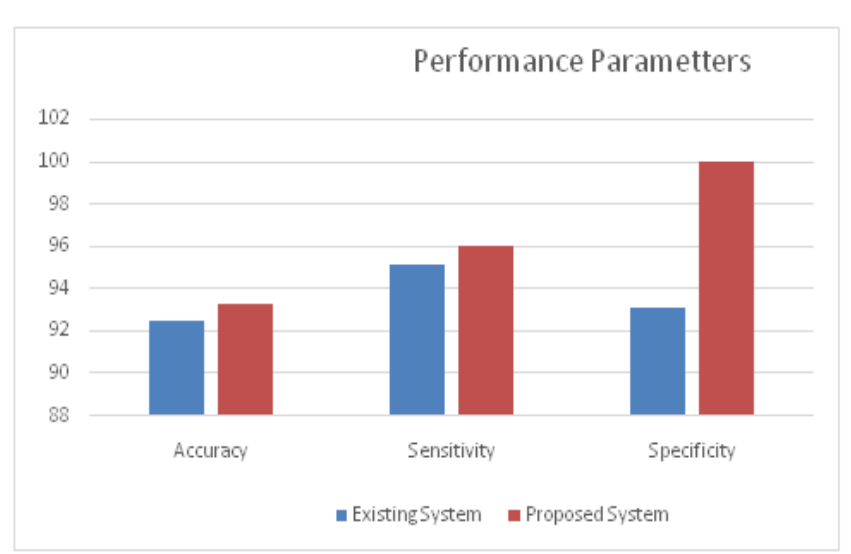

Fig. 6: Comparison of existing and proposed system based on different performance parameters.

\section{Conclusion}

The existence of arrhythmia is reflected in morphology of ECG signal. According to the report by the United Nations in 2015 , the world is confronting an aging population. So, there is need to design an efficient and robust automated computer aided system which accurately detects the various types of arrhythmia. Arrhythmia is characterized as the abnormal vibrations of the heart pulses which can be harmful or not. In proposed system we are going to develop a deep learning 
method to automatically detect arrhythmia and classify them in four classes as Normal, atrial fibrillation, atrial flutter, and ventricular fibrillation. For that purpose, we used modified version of CNN called as a faster R-CNN where R stands for Region. It has Region Proposal Networks (RPNs) for generation of effective and accurate region. In R-CNN, CNN used to concentrate on one region at a time since that way impedance is limited since it is normal that just a single object of interest will influence in a given region. Faster $\mathrm{RCNN}$ has an object detection algorithm itself that eliminates the use of selective search algorithm so it reduces the computation time and gives more precise output. Deep learning is the most powerful way for cardiac abnormality detection and more research is needed in this area.

\section{ACKNOWLEDGMENT}

I would sincerely like to thank my guide Prof. Santosh kumar, Computer Engineering, SITRC, Nashik for his guidance, encouragement and the interest shown in this project by timely suggestions in this work. His expert suggestion and scholarly feedback had greatly enhanced the effectiveness of this work. I would also thank to our head of department Prof. (Dr.) Amol D. Potgantwar, Computer Engineering, SITRC, Nashik for his great support and guidance.

\section{REFERENCES}

[1] U. Rajendra Acharya, Yuki Hagiwara, Oh Shu Lih, Jen Hong Tan, Muhammad Adam, Hamido Fujita," Automated detection of arrhythmias using different intervals of tachycardia ECG segments with convolutional neural network, ELSEVIER, Information Sciences 405, no. 81-90, 2017.

[2] Fernando, Oliver, Marco, Adam, De-Maarten, V., "Comparing feature based classifiers and convolutional neural networks to detect arrhythmia from short segments of ECG', In Proceedings of the Conference on Computing in Cardiology (CinC), Rennes, France, 24-27 September 2017; IEEE: Piscataway, NJ, USA, 2017.

[3] Shadi, G., Mostafa, A., Nasimalsadat, M., Kamran, K., Ali, G., "Atrial fibrillation detection using feature based algorithm and deep conventional neural network", In Proceedings of the Conference on

\section{AUTHORS PROFILE}

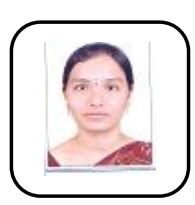

Miss. Swati Dilip Thakare, PG Student, Computer Engineering Department, SITRC, Nashik
Computing in Cardiology (CinC), Rennes, France, 24-27 September 2017; IEEE: Piscataway, NJ, USA, 2017.

[4] National Institute on Aging turning discovery into health. Global health and aging. Assessing the costs of aging and health care. https://www.nia.nih.gov/research/publication/global-health-andaging/ assessing-costsaging-and-health-care.(Last accessed: 24 February 2017).

[5] Acharya, U.R.; Fujita, H., Adam, M., Oh, S.L., Tan, J.H., Sudarshan, V.K., Koh, J.E.W., "Automated characterization of arrhythmias using nonlinear features from tachycardia ECG beats", In Proceedings of the IEEE International Conference on Systems, Man, and Cybernetics (SMC), Budapest, Hungary, 9-12 October 2016; IEEE:Piscataway, NJ, USA, 2016

[6] I. S. Siva Rao, T. Srinivasa Rao, "Performance Identification of Different Heart Diseases Based On Neural Network Classification", International Journal of Applied Engineering Research ISSN 0973-4562 Volume 11, Number 6, pp 3859- 3864, 2016.

[7] M. J. J. P. van Grinsven, B. van Ginneken, C. B. Hoyng, T. Theelen, C. I. Snchez., "Fast convolutional neural network training using selective data sampling: application to haemorrhage detection in color fundus images", IEEE Transactions on Medical Imaging 35(5): 1273-1284, 2016.

[8] Nilanon, T., Yao, J., Hao, J., Purushotam, S., Liu, Y., "Normal/abnormal heart recordings classification by using convolutional neural network", In Proceedings of the IEEE Conference on Computing in Cardiology Conference (CinC), Vancouver, BC, Canada, 11-14 September 2016; IEEE: Piscataway, NJ, USA, 2016, pp. 585-588.

[9] Salam, A.K.; Srilakshmi, G. "An algorithm for ECG analysis of arrhythmia detection", In Proceedings of the IEEE International Conference on Electrical, Computer and Communication Technologies (ICECCT), Coimbatore, India, 5-7 March 2015; IEEE: Piscataway, NJ, USA, 2015; pp. 1-6

[10] Debbal, S.M., "Model of differentiation between normal and abnormal heart sounds in using the discrete wavelet transform", J. Med. Bioeng. 2014, 3, 5-11.

[11] Indu Saini , Dilbag Singh , Arun Khosla, QRS detection using K-Nearest Neighbor algorithm(KNN) and evaluation on standard ECG databases, JOAR, 2013.

[12] G. V. Chow, J. E. Marine, J. L. Fleg., "Epidemiology of arrhythmias and conduction disorders in older adults", Clinics in Geriatric Medicine 28(4): 539-553, 2012.

[13] Yakup Kutlu, DamlaKuntalp, A Multi-Stage Automatic Arrhythmia Recognition And Classification System, ELSEVIER, Computers in Biology and Medicine 41 (2011) 3745.

[14] Moody, G.B.; Mark, R.G., "The impact of the MIT-BIH arrhythmia database". IEEE Eng. Med. Biol. Mag. 2001, 20, 45-50. [CrossRef] [PubMed]

Prof. Santosh Kumar, currently working as Assistant Professor in $\overbrace{\text { Computer Engineering Department, SITRC, Nashik. }}$ 